\title{
IVD.I5 - ELISA assays utilizing aptamers for the detection of the NS5 protein of Zika virus
}

Alessandra Alves Abalo ${ }^{1 *}$; Ana Paula Corrêa Argondizzo ${ }^{1}$; Liliane Monteiro de Morais ${ }^{1}$; Ethel Valdez $^{2}$; Dilson Silva ${ }^{1}$; Sotiris Missailidis ${ }^{1}$.

1Fiocruz/Bio-Manguinhos;

2UERJ - Universidade Estadual do Rio de Janeiro.

Introduction: The infection caused by the flavivirus Zika (ZIKV) remained as a tropical neglected disease until November 2015, when cases of microcephaly in infants born after a ZIKV epidemic, together with an increase in cases of Guillain-Barré syndrome and other neurological disorders were associated with the ZIKV infection. Nonstructural protein 5 (NS5) is essential for replication of the genome, whose RdRp domain synthesizes viral RNA through a de novo synthetic mechanism. Aptamers are single stranded DNA (or RNA) molecules, which bind to a wide range of ligands with high specificity and affinity, and can be used as relevant molecular tools.

Objective: to evaluate the sensitivity and specificity of previously selected ssDNA aptamers, capable of binding the $\mathrm{N}$-terminal portion (483-718 aa) of the recombinant ZIKV NS5 protein (rNS5z) in enzyme-linked immunosorbent assays (ELISA).

Methodology: Five 5'-amino C6-modified (-NH2) aptamers (B03, 20A, A07, C03 and H06) $(0.1 \mu \mathrm{M})$ were tested for capture of $\mathrm{rNS}_{5 \mathrm{z}}(4 \mu \mathrm{g} / \mathrm{mL})$ using ELISA. Mouse serum containing polyclonal antibodies (1:1600 dilution) against $\mathrm{rNS}_{5} \mathrm{z}$ was used for protein detection in modified 96-well plates (activated maleic anhydride amino-reactive) covered by $5^{\prime}-\mathrm{NH}_{2}$ modified aptamers, according to the manufacturer's specification. Anti-IgG antibody (mouse) conjugated to streptavidin-HRP (revealed with TMB) was used for colorimetric reaction, which was discontinued using $1 \mathrm{~N} \mathrm{HCl}$. Optical density reading (O.D.) was performed on a plate reader using wavelength of $450 \mathrm{~nm}$. Human serum albumin (HSA) in PBS and human serum spiked with $\mathrm{rNS} 5 \mathrm{z}$ were used as controls.

Results: The ELISA assays confirmed the detection of rNS5z protein in buffer solution by the five aptamers tested, with O.D. of 1,512 \pm 0,012 (mean \pm standard deviation) and 1,698 \pm 0,005 for aptamers B03 and 20A, respectively. Very similar O.D. values were obtained for the other aptamers tested. These results also showed statistically significant values $(\mathrm{p}<0.001)$ in relation to O.D. observed when using the preimmune serum as a negative control of the reaction, which were $0.08 \pm 0.012$ and $0.073 \pm 0.030$, respectively for the aptamers B03 and 20A. The selectivity of these aptamers was evaluated by adding $6 \mu \mathrm{M}$ human serum albumin (HSA) to the $\mathrm{rNS} 5 \mathrm{z}$ protein solution, and preliminary results indicate that the interaction of aptamers to the target protein did not change significantly $(1,556 \pm 0,033$ and 1,680 $\pm 0,055$ for aptamers B03 and 20A, respectively) upon addition of HSA at physiological levels. Interference of other human serum components was also investigated, using human serum spiked with rNS5z.

Conclusion: Our results indicate that these aptamers could be of value as diagnostic tools for the identification of the ZIKV NS5 protein during the acute infection period.

Keywords: Aptamers; NS5; Zika Virus 